\section{Sobrevida das leucemias linfoides agudas em crianças no Município de São Paulo, Brasil}

\author{
Survival from acute lymphocytic leukemia in \\ children in the city of São Paulo, Brazil
}

\section{Supervivencia de niños con leucemias linfoides agudas, municipio de São Paulo, Brasil}

Franciane Figueiredo da Silva 1

Maria do Rosário Dias de Oliveira Latorre 2

doi: 10.1590/0102-311X00008019

\section{Resumo}

Este estudo visa analisar as taxas de sobrevida da leucemia linfoide aguda na infância no Município de São Paulo, Brasil, no período de 1997 a 2013, segundo sexo, faixa etária e região administrativa. Trata-se de um estudo longitudinal para calcular as taxas de sobrevida. Os dados dos casos novos de leucemia na faixa etária de 0 a 14 anos foram coletados na base de dados do Registro de Câncer de Base Populacional do Município de São Paulo. Os dados populacionais e de mortalidade foram obtidos do Departamento de Informática do SUS. Foram incluídas crianças de 0 a 14 anos, diagnosticadas com leucemia no período de 1997 a 2013, residentes no Município de São Paulo. As variáveis analisadas foram sexo, faixa etária e Coordenadorias Regionais de Saúde. Na análise de sobrevida, foi calculado o estimador produto-limite de Kaplan-Meier, foram comparadas as curvas de sobrevida pelo teste de log-rank e ajustou-se o modelo de riscos proporcionais de Cox. A maioria estimada dos casos novos eram do sexo masculino, de 0 a 4 anos de idade, diagnosticados entre 2000 a 2004 e residentes na regional Sudeste do Município de São Paulo. A taxa de incidência geral estimada foi de 34,7 casos novos por 1 milhão, e a taxa de mortalidade foi de 9,0 óbitos por 1 milhão. A probabilidade geral de sobrevida por leucemia linfoide aguda em crianças em até 60 meses foi de 68\%. Houve diferença estatisticamente significativa segundo década diagnóstica $(p=0,026)$, especificamente, ocorreu piora das taxas de sobrevida ao longo dos anos, sendo que o pior prognóstico foi nos anos de 2005-2009.

Leucemia; Criança; Incidência; Mortalidade; Análise de Sobrevida

\section{Correspondência}

F. F. Silva

Rua Desengano s/n, Vila Velha, ES 29112-431, Brasil.

francianefigueiredo@gmail.com

1 Universidade Federal do Espirito Santo, Vitória, Brasil. 2 Faculdade de Saúde Pública, Universidade de São Paulo, São Paulo, Brasil. 


\section{Introdução}

As leucemias linfoides agudas (LLA) representam 26,8\% dos cânceres infantis e 78,6\% de todas as leucemias. É mais comum em meninos, sendo que a razão de sexos (masculino/feminino) é de 1:3, e boa parte ocorre entre 2 e 9 anos de idade 1 .

Pesquisa realizada pela Sociedade Americana de Câncer, com dados do programa Surveillance, Epidemiology, and End Results (SEER), mostra que a taxa de sobrevida pediátrica em 5 anos, considerando pacientes de até 19 anos de idade, para LLA, passou de 57\%, no período de 1975-1979, para 90\%, no período de 2003-20092.

Recentes pesquisas mostram que países de alta renda, como Canadá, Reino Unido, Bélgica, Dinamarca, Alemanha, Suíça e Holanda, possuem taxa de sobrevida de LLA em crianças superior a 90\%, nos anos de 2010 a 20143.

Há uma lacuna na literatura brasileira sobre estudos de base populacional sobre a leucemia em crianças. Poucos trabalhos dão ênfase aos indicadores epidemiológicos da leucemia infantil, de forma específica. Por exemplo, a taxa de sobrevida populacional desse tipo de câncer infantil é pouco explorada, devido à dificuldade existente em diversos registros de câncer de base populacional do Brasil. A grande maioria dos trabalhos se refere a características de grupos específicos hospitalares.

Dessa forma, este trabalho visa analisar as taxas de sobrevida da LLA na infância no Município de São Paulo, no período de 1997 a 2013, segundo sexo, faixa etária e região administrativa.

\section{Métodos}

Trata-se de um estudo do tipo coorte, observacional. Os dados dos casos novos de leucemia na faixa etária de 0 a 14 anos foram coletados na base de dados do Registro de Câncer de Base Populacional do Município de São Paulo (RCBP-SP). Os dados populacionais e de mortalidade foram obtidos do Departamento de Informática do SUS (DATASUS; http://www2.datasus.gov.br/DATASUS/index. php?area=02, acessado em 12/Jun/2017). Foram incluídas crianças de 0 a 14 anos, diagnosticadas com leucemia (códigos CID-10 entre C91 e C95; https://www.cid10.com.br/, acessado em 06/Mar/2017), no período de 1997 a 2013, residentes no Município de São Paulo. Foram selecionados os pacientes cujo diagnóstico compreendia os códigos de morfologia: 9835, 9836, 9837, 9823, 9826, 9832, 9833, 9940, 9827, 9831, 9834, 9948 e 9820. Foram calculadas as taxas brutas e padronizadas, tanto para incidência quanto para mortalidade.

As taxas foram padronizadas por idade (age-standardized rate - ASR), considerando, como população padrão, a população mundial proposta por Segi e modificada por Doll et al. ${ }^{4}$, para que não ocorresse interferência da estrutura etária, permitindo, assim, a comparação dessas taxas com as taxas de outros lugares e ao longo do período analisado. As taxas foram calculadas segundo sexo (masculino e feminino), faixa etária ( 0 a 4 anos, 5 a 9 anos, 10 a 14 anos) e Coordenadorias Regionais de Saúde (Centro, Leste, Norte, Oeste, Sudeste e Sul).

O tempo de sobrevida foi calculado pela diferença entre a data do primeiro diagnóstico e a data do óbito ou data da última informação. Na análise de sobrevida, o tempo de acompanhamento foi truncado em 60 meses.

Para estimar a função de sobrevivência, foi adotado, neste estudo, o estimador não paramétrico produto-limite de Kaplan-Meier, que também é conhecido por estimador produto-limite. O teste de log-rank foi utilizado para comparação das curvas de sobrevida. Foi ajustado o modelo de riscos proporcionais de Cox, ou modelo de Cox, ou regressão de Cox 5 . O modelo tem a suposição básica que os riscos são proporcionais. A violação dessa suposição pode acarretar sérios vieses de estimação dos coeficientes do modelo, e essa suposição foi verificada pela análise de resíduos de Schoenfeld e por meio dos gráficos do $\ln (-\ln (\mathrm{S}(\mathrm{t}))) 6$.

Esta pesquisa foi encaminhada ao Comitê de Ética em Pesquisa da Faculdade de Saúde Pública da Universidade de São Paulo, por meio do sistema Plataforma Brasil, e, posteriormente, foi aprovada sob o número 1.885.857. Foi autorizada a obtenção dos dados do RCBP-SP, pelo Conselho Técnico Administrativo, e a base de dados fornecida não continha a identificação dos pacientes. O programa utilizado foi o Stata 11.2 (https://www.stata.com). 


\section{Resultados}

Foram contabilizados 1.403 casos novos e 382 óbitos de LLA em crianças no Município de São Paulo. A Tabela 1 mostra os resultados descritivos das taxas de incidência e mortalidade das LLA. Para os casos novos, a maioria foi diagnosticada entre 2000 a 2004, de 0 a 4 anos, do sexo masculino e residentes na regional Sudeste, que é uma região com melhores condições de vida. A maioria dos óbitos ocorreu no período de 2005 a 2009, eram crianças de 5 a 9 anos, do sexo masculino e residentes na regional Norte, que tem índices de qualidade de vida piores do que os da regional Sudeste. A taxa de incidência geral foi de 34,7 casos novos por 1 milhão, e a taxa de mortalidade foi de 9,0 óbitos por 1 milhão.

$\mathrm{Na}$ análise de sobrevida, foram contabilizados 1.160 casos incidentes de LLA, e, desses, 355 foram a óbito. A Tabela 2 mostra os resultados das taxas de sobrevida acumulada segundo as variáveis sob estudo. A probabilidade de sobrevida acumulada em 60 meses por LLA em crianças foi de $68 \%$. Houve diferença estatisticamente significativa segundo década diagnóstica $(\mathrm{p}=0,026$; Figura 1). Não houve diferença estatisticamente significativa segundo faixa etária $(\mathrm{p}=0,484)$, sexo $(\mathrm{p}=0,060)$ e Coordenadoria Regional de Saúde ( $\mathrm{p}=0,338)$.

Os resultados obtidos na Tabela 3 mostram que, independente das demais variáveis, o período de diagnóstico de 2005-2009 teve pior prognóstico, quando comparado ao período de 1997-1999 $($ p global $=0,0213)$.

\section{Tabela 1}

Número e percentual de casos novos e óbitos por leucemias em crianças, de 0 a 14 anos, e suas respectivas taxas, no período de 1997 a 2013 , no Município de São Paulo, Brasil.

\begin{tabular}{|c|c|c|c|c|c|c|}
\hline \multirow[t]{2}{*}{ Variáveis/Categorias } & \multicolumn{3}{|c|}{ Casos novos } & \multicolumn{3}{|c|}{ Óbitos } \\
\hline & $\mathbf{n}$ & $\%$ & Taxa de incidência * & $\mathbf{n}$ & $\%$ & Taxa de mortalidade * \\
\hline \multicolumn{7}{|l|}{ Períodos } \\
\hline 1997-1999 & 334 & 23,8 & 42,9 & 68 & 17,8 & 8,5 \\
\hline $2000-2004$ & 459 & 32,7 & 37,6 & 119 & 31,2 & 9,3 \\
\hline $2005-2009$ & 373 & 26,6 & 32,5 & 121 & 31,7 & 9,8 \\
\hline $2010-2013$ & 237 & 16,9 & 26,6 & 74 & 19,4 & 8,0 \\
\hline \multicolumn{7}{|l|}{ Faixa etária (em anos) ** } \\
\hline $0-4$ & 606 & 43,2 & 44,4 & 97 & 25,4 & 7,1 \\
\hline $5-9$ & 447 & 31,9 & 33,1 & 158 & 41,4 & 11,7 \\
\hline $10-14$ & 350 & 24,9 & 23,6 & 127 & 33,2 & 8,6 \\
\hline \multicolumn{7}{|l|}{ Sexo } \\
\hline Masculino & 805 & 57,4 & 39,3 & 226 & 59,2 & 10,6 \\
\hline Feminino & 598 & 42,6 & 30,0 & 156 & 40,8 & 7,4 \\
\hline \multicolumn{7}{|c|}{ Coordenadoria Regional de Saúde } \\
\hline Centro & 58 & 4,4 & 60,8 & 14 & 3,7 & 14,5 \\
\hline Leste & 269 & 20,5 & 26,2 & 76 & 20,1 & 7,0 \\
\hline Norte & 270 & 20,6 & 33,8 & 99 & 26,2 & 11,8 \\
\hline Oeste & 106 & 8,1 & 38,1 & 25 & 6,6 & 8,5 \\
\hline Sudeste & 308 & 23,5 & 37,0 & 77 & 20,4 & 8,8 \\
\hline Sul & 299 & 22,8 & 29,7 & 87 & 23,0 & 8,2 \\
\hline Ignorado & 93 & - & - & 4 & - & - \\
\hline Total & 1.403 & 100,0 & 34,7 & 382 & 100,0 & 9,0 \\
\hline
\end{tabular}

* Taxas por 1 milhão;

** Taxa bruta. As demais taxas são padronizadas por idade. 


\section{Tabela 2}

Cálculo da tábua de vida atuarial pelo estimador produto-limite de Kaplan-Meier, para os casos incidentes de leucemia linfoide aguda em crianças de 0 a 14 anos, no Município de São Paulo, Brasil.

\begin{tabular}{|c|c|c|c|c|c|}
\hline \multirow[t]{2}{*}{ Variável/Categorias } & \multirow[t]{2}{*}{$\mathbf{n}$} & \multicolumn{3}{|c|}{ Sobrevida acumulada } & \multirow[t]{2}{*}{ Log-rank (valor de $\mathrm{p})$} \\
\hline & & $\begin{array}{c}12 \\
\text { meses }\end{array}$ & $\begin{array}{c}36 \\
\text { meses }\end{array}$ & $\begin{array}{c}60 \\
\text { meses }\end{array}$ & \\
\hline Períodos & & & & & 0,026 \\
\hline 1997-1999 & 309 & 0,8896 & 0,7930 & 0,7437 & \\
\hline $2000-2004$ & 426 & 0,8418 & 0,7086 & 0,6564 & \\
\hline 2005-2009 & 363 & 0,8594 & 0,6950 & 0,6357 & \\
\hline $2010-2013$ & 62 & 0,9001 & 0,7716 & 0,6949 & \\
\hline Faixa etária (anos) & & & & & 0,484 \\
\hline $0-4$ & 524 & 0,8849 & 0,7467 & 0,6878 & \\
\hline $5-9$ & 356 & 0,8656 & 0,7336 & 0,6733 & \\
\hline $10-14$ & 280 & 0,8205 & 0,6993 & 0,6577 & \\
\hline Sexo & & & & & 0,062 \\
\hline Feminino & 487 & 0,8699 & 0,7541 & 0,7094 & \\
\hline Masculino & 673 & 0,8586 & 0,7147 & 0,6525 & \\
\hline Coordenadoria Regional de Saúde * & & & & & 0,308 \\
\hline Centro & 48 & 0,8542 & 0,7479 & 0,6784 & \\
\hline Leste & 207 & 0,8621 & 0,7469 & 0,6918 & \\
\hline Norte & 227 & 0,8205 & 0,6887 & 0,6010 & \\
\hline Oeste & 93 & 0,8908 & 0,7080 & 0,6726 & \\
\hline Sudeste & 257 & 0,8685 & 0,7233 & 0,6723 & \\
\hline Sul & 253 & 0,8764 & 0,7325 & 0,6971 & \\
\hline Total & 1.160 & 0,8633 & 0,7312 & 0,6763 & \\
\hline
\end{tabular}

* Nesta análise foram excluídos 75 casos em que não havia referência da Coordenadoria a qual pertenciam.

\section{Discussão}

A taxa de incidência por LLA no Município de São Paulo (37,4 casos novos por 1 milhão) é semelhante às taxas de alguns países da América Latina e Caribe, bem como do Canadá e Estados Unidos 7. Porém, as taxas de mortalidade são altas quando comparadas às taxas dos Estados Unidos 8. Sabe-se também que as taxas de sobrevida de outros países aumentaram ao longo dos anos, diminuindo, assim, as taxas de mortalidade.

A taxa de sobrevida após 60 meses no Município de São Paulo para leucemia foi de 67,63\%. Esse valor é próximo aos encontrados em países da América Central e do Sul, em que a menor taxa de sobrevida relativa em 5 anos para LLA foi de 62,6\% no Equador, e a maior taxa foi de 80,1\% em Porto Rico, para os anos de 2005 a 2009. Essa taxa ainda é baixa, quando comparada a países como Estados Unidos e Canadá, em que, no mesmo período, as taxas de sobrevida relativa em 5 anos foram de 87,7\% e 90,6\%, respectivamente. Igualmente, na Europa, no mesmo período, as maiores e menores taxas de sobrevida relativa em 5 anos, respectivamente, foram encontradas na Alemanha $(91,8 \%)$ e Estônia $(62,6 \%)^{9}$.

Segundo sexo, as taxas de sobrevida são maiores em meninas que em meninos, respectivamente, $70,94 \%$ e $65,25 \%$. No Canadá, essa diferença na sobrevida das LLA em 5 anos foi encontrada em todos os períodos analisados 10. Estudo realizado na Europa pelo EUROCARE-5 mostrou essa diferença entre a LLA (meninas com 87,6\% e meninos com 85,3\%) 11.

A faixa etária com menor sobrevida em São Paulo foi para crianças com idade entre 10 a 14 anos: 65,77\%. Achados do CONCORD-2 mostrou que a menor taxa de sobrevida para LLA nos Estados 


\section{Figura 1}

Gráfico de sobrevida acumulada de Kaplan-Meier, segundo período de diagnóstico, para os casos incidentes de leucemia linfoide aguda em crianças de 0 a 14 anos, no Município de São Paulo, Brasil.

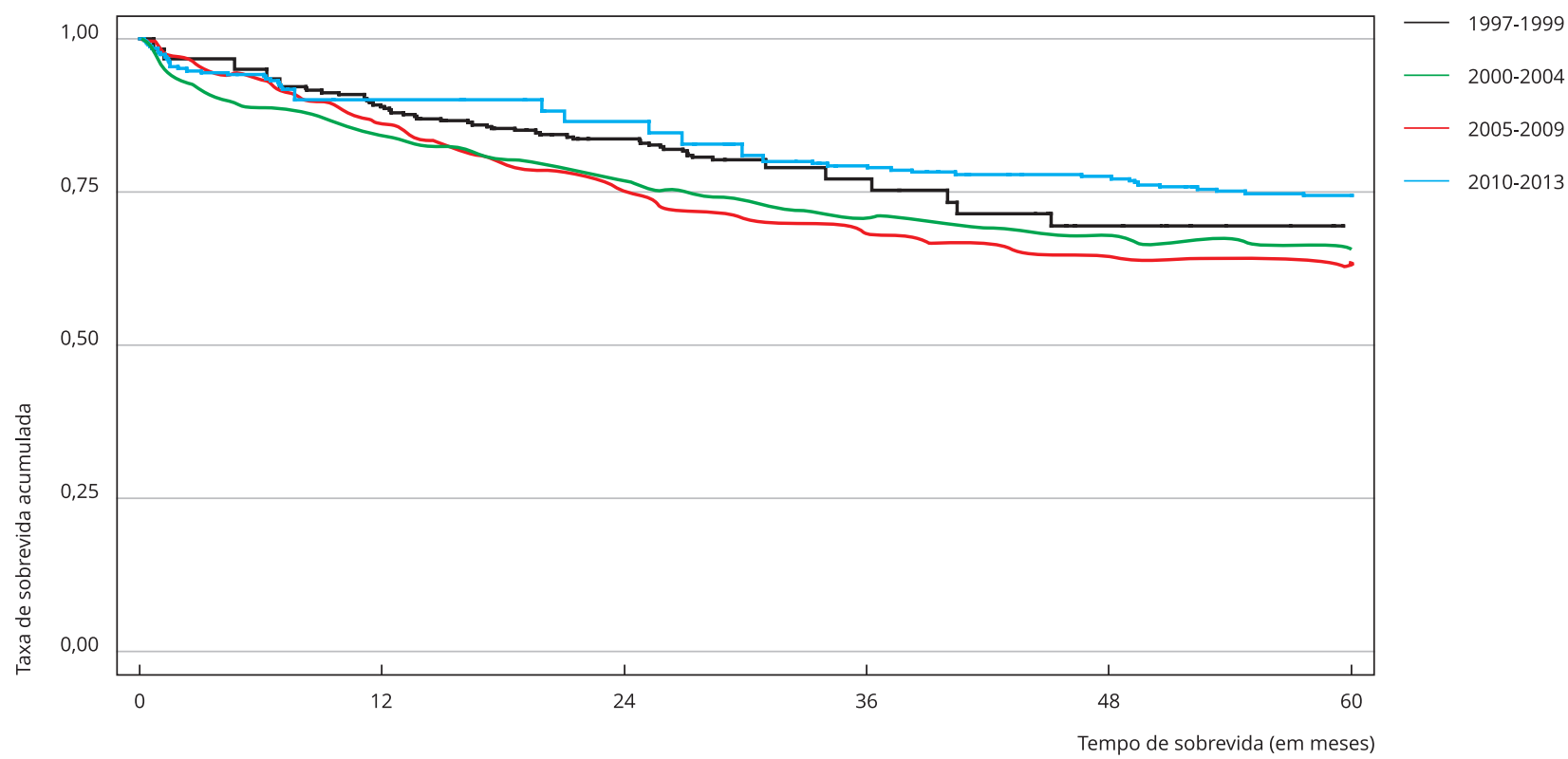

Unidos, de 2004 a 2009, foi para os menores de 1 ano, com 60,1\%, seguidos pelos que a idade está entre 10 a 14 anos, com $81,5 \% 12$.

Em São Paulo, na contramão da tendência global, houve diminuição das taxas de sobrevida por LLA nos períodos estudados. Registros brasileiros mostram essa diminuição, como corroboram com os estudos mundiais 9,13. Além do Brasil, Malásia e Eslovênia apresentaram comportamento semelhante. Na Malásia, a taxa de sobrevida relativa para LLA caiu de 77\%, de 1995 a 1999, para 69\%, em 2005 a 2009, e, na Eslovênia, foi de 83\%, de 1995 a 1999, para 76\%, em 2005 a 20099.

Estudo do CONCORD-2 mostrou a queda das taxas de sobrevida no Brasil de $72 \%$, nos anos de 1995 a 1999, para 66\%, nos anos de 2005 a 2009. Foi indicado que a queda, possivelmente, reflete a organização e o financiamento dos serviços de saúde. A sobrevida foi afetada pela pouca melhoria das estratégias de enfrentamento do câncer em países como o Brasil. Em contrapartida, após mudanças políticas e econômicas em países como Estônia, Lituânia e Alemanha, foram relatadas melhorias nas taxas de sobrevida do câncer 9 .

Um estudo mais recente do CONCORD-3 mostrou que a sobrevida relativa das LLA no Brasil ainda ficava abaixo de 70\%, mesmo após ajustes para o cenário de alta mortalidade infantil. Os autores enfatizam que a letalidade das neoplasias infantis é alta em países de média e baixa renda, onde o acesso e o abandono do tratamento são grandes problemas 3 .

Quando comparadas as baixas taxas de sobrevida brasileiras às taxas de sobrevida de países de alta renda, que, em geral, são altas, sugere-se que existam dificuldades ou desigualdades no acesso e no diagnóstico no Brasil. Isto é, a sobrevida de neoplasias curáveis, como a leucemia em crianças, está intimamente ligada à renda do país ${ }^{14}$. Ações para detecção e tratamento precoce seguem inacessíveis para grande parcela da população dos países em desenvolvimento, tais como o Brasil, embora haja disponibilidade de opções eficazes de tratamento e de baixo custo para alguns cânceres, incluindo a leucemia infantil 15 . 
Tabela 3

Resultado do modelo de riscos proporcionais de Cox, bruto e ajustado, das leucemias linfoides agudas em crianças, no Município de São Paulo, Brasil, de 1997 a 2013.

\begin{tabular}{|c|c|c|c|c|}
\hline Variável/Categorias & HR bruto & IC95\% & HR ajustado & IC95\% \\
\hline \multicolumn{5}{|l|}{ Períodos } \\
\hline 1997-1999 & 1,00 & - & 1,00 & - \\
\hline $2000-2004$ & 1,43 & $1,09-1,89$ & 1,30 & $0,98-1,74$ \\
\hline 2005-2009 & 1,51 & $1,13-2,01$ & 1,43 & $1,06-1,92$ \\
\hline 2010-2013 & 1,18 & $0,70-1,99$ & 1,08 & $0,63-1,87$ \\
\hline \multicolumn{5}{|l|}{ Faixa etária (anos) } \\
\hline $0-4$ & 1,00 & - & 1,00 & - \\
\hline $5-9$ & 1,07 & $0,83-1,36$ & 1,04 & $0,81-1,34$ \\
\hline $10-14$ & 1,17 & $0,91-1,52$ & 1,12 & $0,86-1,46$ \\
\hline \multicolumn{5}{|l|}{ Sexo } \\
\hline Feminino & 1,00 & - & 1,00 & - \\
\hline Masculino & 1,23 & $0,99-1,52$ & 1,19 & $0,96-1,49$ \\
\hline \multicolumn{5}{|c|}{ Coordenadoria Regional de Saúde } \\
\hline Centro & 1,00 & & 1,00 & \\
\hline Leste & 0,98 & $0,55-1,72$ & 0,95 & $0,54-1,67$ \\
\hline Norte & 1,34 & $0,77-2,32$ & 1,35 & $0,78-2,34$ \\
\hline Oeste & 1,04 & $0,56-1,93$ & 1,06 & $0,57-1,98$ \\
\hline Sudeste & 1,04 & $0,60-1,80$ & 1,06 & $0,61-1,85$ \\
\hline Sul & 0,95 & $0,55-1,66$ & 0,97 & $0,55-1,69$ \\
\hline
\end{tabular}

IC95\%: intervalo de 95\% de confiança; HR: hazard ratio.

Sabe-se que o Sistema Único de Saúde (SUS) atende a maior demanda para tratamento de serviços de alta complexidade, como o câncer 16 . E, apesar de obter um parâmetro acima do recomendado pelo Ministério da Saúde em relação aos procedimentos quimioterápicos do total de consultas realizadas, dados mostram que São Paulo obteve uma redução no gasto anual per capita com procedimentos em quimioterapia, sendo consequência da redução ou menor frequência de procedimentos 17.

Estudos mostram que, em Recife (Pernambuco), a taxa de sobrevida em 5 anos foi de $63 \%$, a um custo de USD 16.700 por paciente 18. Em países, tais como os da América do Norte e na Europa Ocidental, onde o tratamento da leucemia em crianças costuma ser mais caro, entre USD 115.858 e USD 163.350 por paciente ${ }^{19}$, a sobrevida também é maior.

Países de baixa e média renda costumam possuir o tratamento em que o custo-efetivo é elevado, segundo critérios da Organização Mundial da Saúde (OMS). Acesso à quimioterapia e custos relacionados à medicação são os principais entraves. As despesas mensais com tratamento de LLA para uma criança ultrapassa em sete vezes a renda per capita mensal em países de baixa renda 20 .

A insuficiência do financiamento do SUS gera obstáculos para alcançar o acesso universal aos serviços de saúde. Com o novo Regime Fiscal, apresentado por meio da Proposta de Emenda à Constituição no 241/2016 (PEC 241), esse cenário é ainda pior. Maiores cortes são esperados, acarretando menor disponibilidade de recursos per capita, afetando, assim, a garantia do direito à saúde 21 .

Com a persistência desse congelamento dos financiamentos em saúde, espera-se uma piora ainda maior das taxas de sobrevida das leucemias infantis nos anos futuros, sendo que essa neoplasia é considerada curável 2,22 , podendo chegar a $83 \%$ de taxa de sobrevida, quando existe cuidado e investimento adequado. 


\section{Colaboradores}

F. F. Silva contribuiu com a concepção do projeto, análise e interpretação dos dados e redação do artigo. M. R. D. O. Latorre contribuiu com a concepção do projeto, interpretação dos dados, redação e revisão crítica.

\section{Informações adicionais}

ORCID: Franciane Figueiredo da Silva (0000-00018125-6582); Maria do Rosário Dias de Oliveira Latorre (0000-0002-5189-3457).

\section{Referências}

1. Kaatsch P. Epidemiology of childhood cancer. Cancer Treat Rev 2010; 36:277-85.

2. Ward E, DeSantis C, Robbins A, Kohler B, Jemal A. Childhood and adolescent cancer statistics, 2014. CA Cancer J Clin 2014; 64:83-103.

3. Allemani C, Matsuda T, Di Carlo V, Harewood R, Matz M, Nikšić M, et al. Global surveillance of trends in cancer survival 2000-14 (CONCORD-3): analysis of individual records for $37,513,025$ patients diagnosed with one of 18 cancers from 322 population-based registries in 71 countries. Lancet 2018; 391:1023-75.

4. Doll R, Payne P, Waterhouse J. Cancer incidence in five countries. Lyon: International Union Against Cancer; 1966.

5. Giolo SR, Colosimo EA. Análise de sobrevivência aplicada. São Paulo: Edgard Blucher; 2006.

6. Carvalho MS, Andreozzi VL, Codeço CT, Barbosa MTS, Shimakura SE. Análise de sobrevida: teoria e aplicações em saúde. Rio de Janeiro: Editora Fiocruz; 2005.

7. Steliarova-Foucher E, Colombet M, Ries L, Hesseling P, Moreno F, Shin H, Stiler C. International incidence of childhood cancer, volume iii (electronic version). http://iicc.iarc.fr/ results/ (acessado em 21/Abr/2017).

8. Smith MA, Altekruse SF, Adamson PC, Reaman GH, Seibel NLJC. Declining childhood and adolescent cancer mortality. Cancer 2014; 120:2497-506.

9. Allemani C, Weir HK, Carreira H, Harewood $\mathrm{R}$, Spika D, Wang XS, et al. Global surveillance of cancer survival 1995-2009: analysis of individual data for $25,676,887$ patients from 279 population-based registries in 67 countries (CONCORD-2). Lancet 2015; 385:977-1010.

10. Ye X, Torabi M, Lix LM, Mahmud SM. Time and spatial trends in lymphoid leukemia and lymphoma incidence and survival among children and adolescents in Manitoba, Canada: 1984-2013. PLoS One 2017; 12:e175701.

11. Gatta G, Botta L, Rossi S, Aareleid T, BielskaLasota M, Clavel J, et al. Childhood cancer survival in Europe 1999-2007: results of Eurocare-5 - a population-based study. Lancet Oncol 2014; 15:35-47.

12. Tai EW, Ward KC, Bonaventure A, Siegel DA, Coleman MP. Survival among children diagnosed with acute lymphoblastic leukemia in the United States, by race and age, 2001 to 2009: findings from the CONCORD-2 study. Cancer 2017; 123 Suppl 24:5178-89.

13. Bonaventure A, Harewood R, Stiller CA, Gatta G, Clavel J, Stefan DC, et al. Worldwide comparison of survival from childhood leukaemia for 1995-2009, by subtype, age, and sex (CONCORD-2): a population-based study of individual data for 89,828 children from 198 registries in 53 countries. Lancet Haematol 2017; 4:e202-17. 
14. Schmidt MI, Duncan BB, Azevedo e Silva G, Menezes AM, Monteiro CA, Barreto SM, et al. Chronic non-communicable diseases in Brazil: burden and current challenges. Lancet 2011; 377:1949-61.

15. Farmer P, Frenk J, Knaul FM, Shulman LN, Alleyne G, Armstrong L, et al. Expansion of cancer care and control in countries of low and middle income: a call to action. Lancet 2010; 376:1186-93.

16. Bahia L, Simmer E, Oliveira DC. Cobertura de planos privados de saúde e doenças crônicas: notas sobre utilização de procedimentos de alto custo. Ciênc Saúde Colet 2004; 9:921-9.

17. Vianna SM, Nunes A, Góes G, Silva JR, Santos RJM. Atenção de alta complexidade no SUS: desigualdades no acesso e financiamento. v. I. Relatório de pesquisa. Brasília: Ministério da Saúde/Instituto de Pesquisa Econômica Aplicada; 2005.

18. Bhakta N, Martiniuk AL, Gupta S, Howard SC. The cost effectiveness of treating paediatric cancer in low-income and middle-income countries: a case-study approach using acute lymphocytic leukaemia in Brazil and Burkitt lymphoma in Malawi. Arch Dis Child 2013; 98:155-60
19. Gupta S, Howard SC, Hunger SP, Antillon FG Metzger ML, Israels T, et al. Treating childhood cancer in low-and middle-income countries. In: Gelband H, Jha P, Sankaranarayanan R, Horton S, editores. Cancer. Washington DC: The World Bank Group; 2015. p. 121-46.

20. Pui CH, Yang JJ, Bhakta N, Rodriguez-Galindo C. Global efforts toward the cure of childhood acute lymphoblastic leukaemia. Lancet Child Adolesc Health 2018; 2:440-54.

21. Vieira FS, Benevides RPS. Os impactos do novo regime fiscal para o financiamento do Sistema Único de Saúde e para a efetivação do direito à saúde no Brasil. Brasília: Instituto de Pesquisa Econômica Aplicada; 2016. (Nota Técnica, 28).

22. DeVita VT, Lawrence TS, Rosenberg SA, DePinho RA, Weinberg RA. Devita, Hellman, and Rosenberg's cancer: principles and practice of oncology. Philadelphia: Wolters Kluwer Health; 2005. 


\section{Abstract}

This study aims to analyze survival rates in acute lymphocytic leukemia in the city of São Paulo, Brazil, from 1997 to 2013, according to sex, age bracket, and administrative region. This was a longitudinal study to calculate survival rates. Data on new cases of leukemia in the age bracket 0 to 14 years of age were collected from the database in the Population-Based Cancer Registry of the city of São Paulo. Population and mortality data were obtained from the Brazilian Health Informatics Department. The sample included children 0 to 14 years of age diagnosed with leukemia from 1997 to 2013, living in the city of São Paulo. The variables were sex, age bracket, and Regional Health Divisions. The survival analysis calculated the Kaplan-Meier product limit estimator and compared the survival curves through the log-rank test, and the Cox proportional risks model was adjusted. The estimated majority of new cases were males, 0 to 4 years of age, diagnosed from 2000 to 2004, and living in the southeast regional division of the city of São Paulo. The estimated overall incidence rate was 34.7 new cases per million, and the mortality rate was 9.0 deaths per million. The overall five-year survival of children from acute lymphocytic leukemia was 68\%. There was a statistically significant difference according to decade of diagnosis $(p=0.026)$, and specifically the survival rates decreased over the years, with the worst prognosis in the years 2005-2009.

Leukemia; Child; Incidence; Mortality; Survival Analysis

\section{Resumen}

El objetivo de este estudio es analizar las tasas de supervivencia de niños con leucemia linfoide aguda en el municipio de São Paulo, Brasil, durante el período de 1997 a 2013, según sexo, franja de edad y región administrativa. Se trata de un estudio longitudinal para calcular las tasas de supervivencia. Los datos de los casos nuevos de leucemia en la franja de edad de 0 a 14 años se recogieron en la base de datos del Registro de Cáncer de Base Poblacional del municipio de São Paulo. Los datos poblacionales y de mortalidad se obtuvieron del Departamento de Informática del Sistema Único de Salud. Se incluyeron niños de 0 a 14 años, diagnosticados con leucemia durante el período de 1997 a 2013, residentes en el municipio de São Paulo. Las variables analizadas fueron sexo, franja de edad y Coordinaciones Regionales de Salud. En el análisis de supervivencia, se calculó el estimador del producto-límite (Kaplan-Meier), se compararon las curvas de supervivencia a través del test de log-rank, y se ajustó el modelo de riesgos proporcionales de Cox. La mayoría estimada de casos nuevos eran de sexo masculino, de 0 a 4 años de edad, diagnosticados entre 2000 a 2004 y residentes en la regional Sudeste del municipio de São Paulo. La tasa de incidencia general estimada fue de 34,7 casos nuevos por 1 millón y la tasa de mortalidad fue de 9,0 óbitos por 1 millón. La probabilidad general de supervivencia por leucemia linfoide aguda en niños de hasta 60 meses fue de un $68 \%$. Hubo una diferencia estadísticamente significativa según la década del diagnóstico $(p=$ 0,026), específicamente, se produjo un empeoramiento de las tasas de supervivencia a lo largo de los años, donde el peor pronóstico fue durante los años de 2005-2009.

Leucemia; Niño; Incidencia; Mortalidad; Análisis de Supervivencia
Recebido em 15/Jan/2019

Versão final reapresentada em 08/Jul/2019

Aprobado em 06/Set/2019 